\title{
ORTHOGONAL BASIS FUNCTIONS OVER THE BINOCULAR PUPIL
}

\author{
Richard J. Mathar
}

Leiden Observatory, Leiden University, Postbus 9513, 2300 RA Leiden, The Netherlands

Received: 2010 March 30; revised and accepted: 2010 June 25

\begin{abstract}
Sets of orthogonal basis functions over circular areas - pupils in optical applications - are known in the literature for the full circle (Zernike or Jacobi polynomials) and the annulus. Here, an orthogonal set is proposed if the area is two non-overlapping circles of equal size. The geometric master parameter is the ratio of the pupil radii over the distance between both circles. Increasingly higher order aberrations - as defined for a virtual larger pupil in which both pupils are embedded - are fed into a Gram-Schmidt orthogonalization to distill one unique set of basis functions. The key effort is to work out the overlap integrals between a full set of primitive basis functions of hyperspherical type centered at the mid-point between both pupils. Constructed from the same primitive basis, the orthogonal Karhunen-Loève modes of spatially filtered Kolmogorov phase screens are computed for this shape of mask. Matrix elements of the covariance matrix - an established intra-circle and a special inter-circle category - are worked out in wavenumber space.
\end{abstract}

Key words: instrumentation: interferometers - methods: numerical - atmospheric effects

\section{OVERVIEW}

Manufacturing schemes of lenses and mirrors inevitably prefer circular crosssections of beams, and the associated description of functions (aberrations) defined across these fields calls for basis functions on this circular support, the best-noted probably being the Zernike functions (Bhatia \& Wolf 1952; Noll 1976; Chong et al. 2003; Prata \& Rusch 1989; Sheppard et al. 2004). Masking a central circular portion of a circular beam leads to annular, ring-shaped regions, for which orthogonal basis sets are also documented (Dai \& Mahajan 2007b; Hou et al. 2006; Mahajan 1981; Wang \& Silva 1980; Swantner \& Lowrey 1980; Mathar 2009).

This work proceeds to define such a basis for a two-beam interferometer, in which the input pupil is a mask of two disconnected circular areas of equal radius (Hu et al. 1989). The layout represents all optical long-baseline interferometers to-date.

We define the area of integration in a global circular coordinate system centered in between the two apertures in Section 2. If general functions defined over these apertures are expanded in a sum of products of powers of the distance to the origin of coordinates by the usual sinusoidal series in the azimuth, all integrals over products of these reduce to a generic integral, summarized in Section 3. The 
lasting value of this article lies in the reduction of two associated integrals. Section 4 proceeds with the re-orthogonalization of the Zernike basis functions - defined over the larger area that encompasses both circles - with respect to the two circular regions that define the binocular pupil.

In the limit of the Fraunhofer diffraction, the imaging properties of the pupil are described by the Fourier Transform - computed in Section 5 - the fringe patterns of image plane recombination (Menut et al. 2008). This parallels the error analysis of the single circular parabolic mirror (Arguijo \& Scholl 2003).

The perturbation of the ideal plane wave phase function by atmospheric turbulence is one application of these concepts, which is studied in the frame work of modal decomposition of Kolmogorov phase screens in Section 6. The bivariate covariance is an operator which also emits orthogonal eigenfunctions. These are preferentially computed in wavenumber space as pointed out by Roddier (1990). Examples are shown where the ratio of the single-telescope diameter over the baseline length is $1 / 2$.

\section{BINOCULAR GEOMETRY}

Orthogonality of functions $f_{k}$ and $f_{l}$ over areas $\Omega$ is defined through their product integrated over the area

$$
\left(f_{k}, f_{l}\right)=\iint f_{k}^{*} f_{l} d \Omega \sim \delta_{k l} .
$$

The star denotes the complex-conjugate of a value. In Cartesian coordinates $\rho_{x}$ and $\rho_{y}$, or circular coordinates with distance $\rho$ to the origin and azimuth $\theta$,

$$
\rho_{x}=\rho \cos \theta ; \quad \rho_{y}=\rho \sin \theta,
$$

the differential is $d \Omega_{R}=d \rho_{x} d \rho_{y}=\rho d \rho d \theta$. This manuscript deals with areas that are the sum of the interior of two circular pupils represented by

$$
\left(\rho_{x} \pm R\right)^{2}+\rho_{y}^{2} \leq r^{2},
$$

where $R$ is half the distance between the two pupil centers, where $2 R$ is the interferometric baseline, and $r$ is each pupil's radius (Figure 1). This transforms the integral operator into a sum over both circles,

$$
\iint d \Omega_{R}=\int_{R-r}^{R+r} \rho d \rho\left[\int_{2 \rho R \cos \theta \leq r^{2}-R^{2}-\rho^{2}} d \theta+\int_{2 \rho R \cos \theta \geq \rho^{2}-r^{2}+R^{2}} d \theta\right] .
$$

Scaling distances in units of $R$,

$$
x \equiv \rho_{x} / R ; \quad y \equiv \rho_{y} / R ; \quad z \equiv \rho / R ; \quad d \Omega_{R}=R^{2} d \Omega,
$$

leaves $q \equiv r / R$ as the essential shape parameter. We only discuss the case without overlap,

$$
0<q \leq 1
$$

The parameter is $q \approx 0.58$ for the Large Binocular Telescope, $q>0.11$ for the Keck Interferometer - a lower bound set by the projected baseline while pointing perpendicular to the baseline - or as small as 0.013 for Auxiliary Telescopes at stations B6 and M0 of the Very Large Telescope Interferometer. 


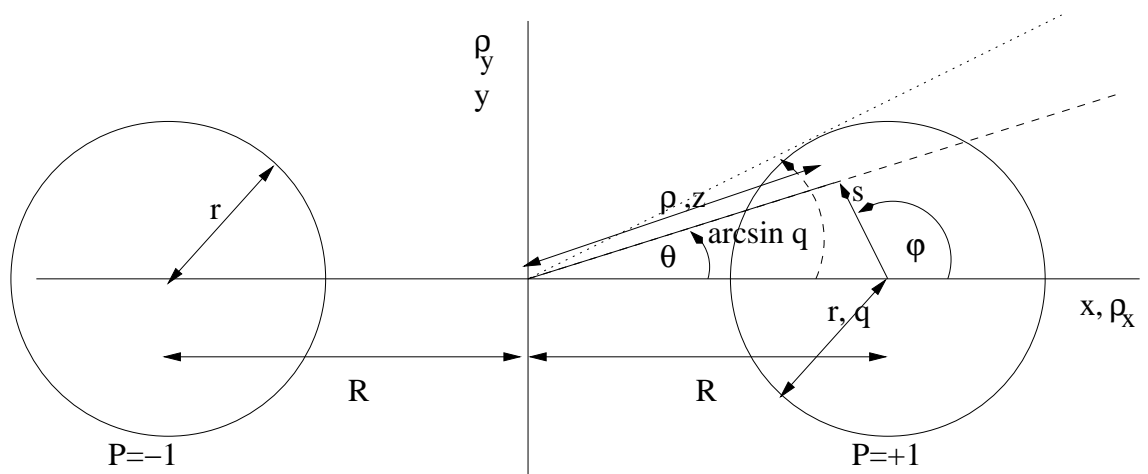

Fig. 1. The two-dimensional area and the Cartesian and polar coordinates covered by the two circular sub-pupils. The center of symmetry and origin of the global circular coordinates is at $x=y=0$. In the first quadrant, the limit $\theta \leq \arcsin q$ of integration is given by the tangent of a radial vector on the pupil with index $P=+1$ along the dotted line.

The range of radial distances inside the circle centered at $x=+1$ is

$$
z=\cos \theta \pm \sqrt{q^{2}-\sin ^{2} \theta}
$$

for some fixed direction $\theta$. The dashed line in Figure 1 illustrates how the radial vector into direction $\theta$ intersects the front and rear side of the circle $P=+1$, associated with the two signs. For the opposite circle centered at $x=-1$ the range is

$$
z=-\cos \theta \pm \sqrt{q^{2}-\sin ^{2} \theta} .
$$

In each of the two equations, the lower sign refers to the intersection with the circle rim that is closer to the origin, the upper sign to the intersection with the farther one.

\section{GENERIC INTEGRAL}

Performing overlap integrals (1) in analytical terms over the area described above is at the heart of this work; since this will be based on spanning the functional space with "primitive" basis functions $p$ of the kind

$$
p_{k} \equiv p_{n_{k}, m_{k}}(z, \theta) \equiv z^{n_{k}} e^{i m_{k} \theta},
$$

the fundamental integral reads

$$
A_{n, m}(q) \equiv \int_{|\sin \theta| \leq q} d \theta \int_{\substack{2 z \cos \theta \leq q^{2}-z^{2}-1 \\ 2 z \cos \theta \geq z^{2}-q^{2}+1}} z d z z^{n} e^{i m \theta}, \quad n, m=0,1,2, \ldots
$$

$i$ is the imaginary unit, $m$ is the azimuthal frequency, and $n$ is the power to complete the radial basis. With a sign tag $P$ defined for both circles,

$$
P \equiv \begin{cases}+1, & |\theta|<\pi / 2 \\ -1, & \pi / 2 \leq|\theta| \leq \pi\end{cases}
$$


the integration over $z^{n+1}$ may be executed,

$$
\begin{aligned}
(n+2) A_{n, m}(q)=\int_{|\sin \theta| \leq q} d \theta\left[\left(P \cos \theta+\sqrt{q^{2}-\sin ^{2} \theta}\right)^{n+2}\right. \\
\left.-\left(P \cos \theta-\sqrt{q^{2}-\sin ^{2} \theta}\right)^{n+2}\right] e^{i m \theta} .
\end{aligned}
$$

The contribution from the imaginary part $\sim \sin (m \theta)$ vanishes; so $e^{i m \theta}$ can be replaced by $\cos (m \theta)$ in this equation. Considering the coordinate transformation $y \leftrightarrow-y$ or $\theta \leftrightarrow-\theta$, the integral over all four quadrants can be reduced to an integral over the first and second quadrant and a factor of 2. Considering also the variable transformation $\theta \leftrightarrow \pi-\theta$, the parities of the Chebyshev polynomial $T_{m}(\cos \theta)=\cos (m \theta)$, the parities of $\cos \theta$ and of $P$, this vanishes for odd $m$ and reduces to an integral over the first quadrant for even $m$,

$$
\begin{aligned}
\frac{(n+2) A_{n, m}(q)}{4}= & \int_{\substack{\sin \theta \leq q \\
0 \leq \theta \leq \pi / 2}} d \theta\left[\left(\cos \theta+\sqrt{q^{2}-\sin ^{2} \theta}\right)^{n+2}\right. \\
& \left.-\left(\cos \theta-\sqrt{q^{2}-\sin ^{2} \theta}\right)^{n+2}\right] \cos (m \theta) .
\end{aligned}
$$

If we define

$$
B_{n+2, m}^{ \pm}(q) \equiv \int_{0}^{\arcsin q} d \theta\left(\cos \theta \pm \sqrt{q^{2}-\sin ^{2} \theta}\right)^{n+2} T_{m}(\cos \theta),
$$

this can be rephrased

$$
\frac{(n+2) A_{n, m}(q)}{4}= \begin{cases}0 & , m=1,3,5,7, \ldots \\ B_{n+2, m}^{+}(q)-B_{n+2, m}^{-}(q) & , m=0,2,4,6, \ldots\end{cases}
$$

Table 1 illustrates the series expansions of $A_{n, m}(q)$ obtained in Appendix A.

To lowest order in $q, A_{n, m}$ equals $2 \pi q^{2}$, the total area of both circles. This is expected, because for circles far away from their common origin at $z=0$, the relative strength of the variation introduced by the power $n$ and by the modulation $\propto \cos (m \theta)$ of the function in the integral kernel loses importance.

\section{GRAM-SCHMIDT ORTHOGONALIZATION}

\subsection{Procedure}

The integral evaluated in Section 3 allows to calculate the overlap integral (inner product) between any two functions expressed as linear combinations of basis functions $p$ of the form (9), because the overlap between any two of these is

$$
\left(p_{k}, p_{l}\right) \equiv \iint p_{k}^{*} p_{l} d \Omega=\iint z^{n_{k}} e^{-i m_{k} \theta} z^{n_{l}} e^{i m_{l} \theta} z d z d \theta=A_{n_{k}+n_{l},\left|m_{k}-m_{l}\right|}(q) .
$$


Table 1. Examples of series expansions of $A_{n, m}(q) / \pi$. These are polynomials in $q^{2}$ if $n-m$ is even, else shown as truncated power series.

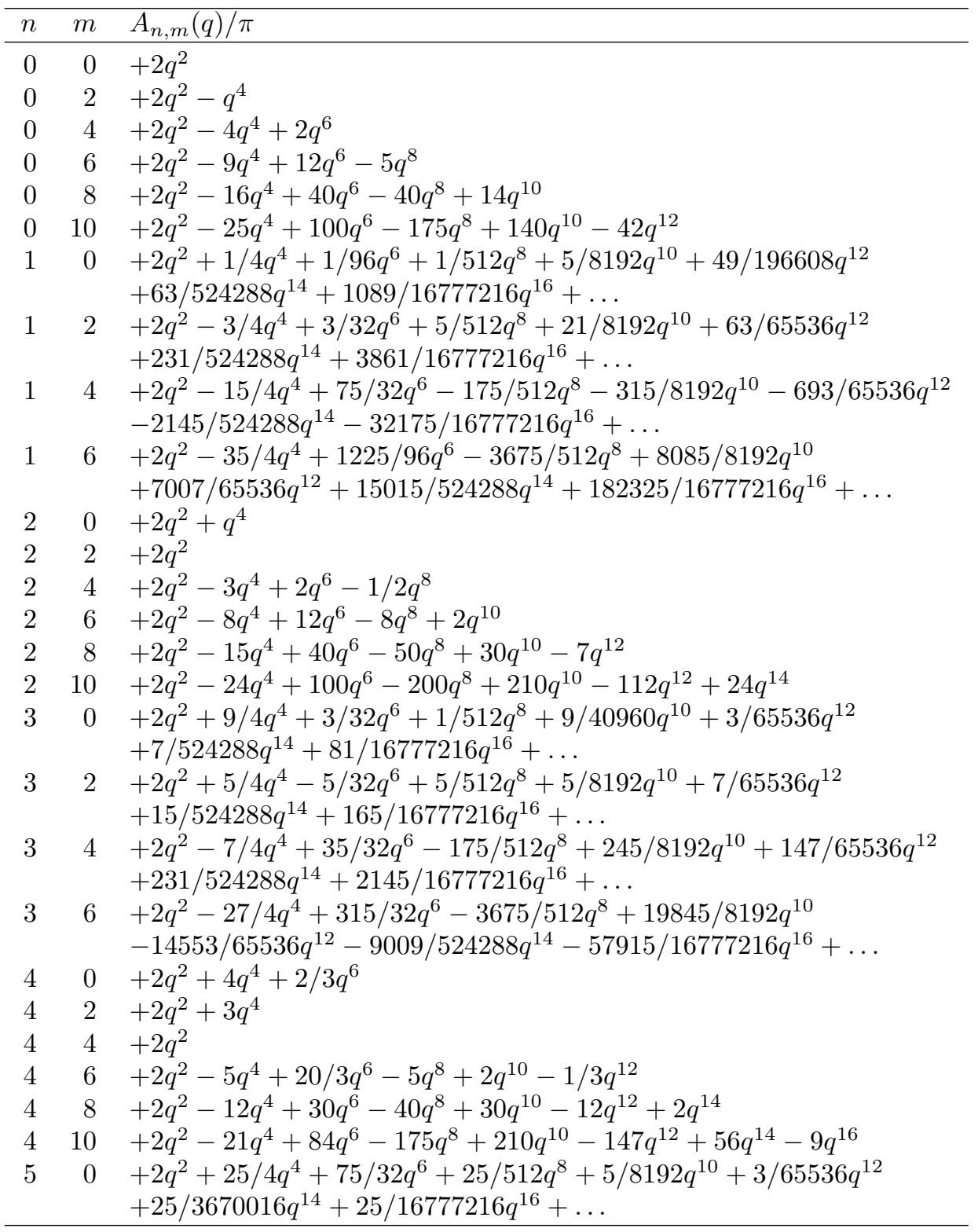


The quickest, obvious way of obtaining some orthogonal basis set from any set of contracted primitive basis function is to diagonalize the overlap matrix containing all the overlap integrals between pairs of these basis functions (Dai \& Mahajan 2007a). To end up with some standardization of these orthogonal bases, we use the Gram-Schmidt procedure, which builds this set $\left\{f_{k}\right\}$ incrementally. At each step, the next basis function $f_{k+1}$ is written as

$$
f_{k+1}(z, \theta)=\beta_{k+1}\left[g_{k+1}(z, \theta)+\sum_{l=1}^{k} \gamma_{k+1, l} f_{l}(z, \theta)\right],
$$

given a seed function $g_{k+1}(z, \theta)$ plus the $f_{l}$ known from the earlier steps. Essentially, the projections of the seed along all earlier directions are subtracted, and the residual is normalized to unity. The request of orthogonality

$$
\iint f_{k}^{*} f_{l} d \Omega=\left(f_{k}, f_{l}\right)=\delta_{k l}, \quad k, l=1, \ldots k+1
$$

means the projection coefficients $\gamma$ can be calculated from the $k$ overlaps between the seed and the earlier basis functions,

$$
\gamma_{k+1, l}=-\left(g_{k+1}, f_{l}\right), \quad l=1, \ldots k .
$$

The normalization $\beta_{k+1}$ is finally computed from the square of the seed and the sum over the squared $\gamma$,

$$
1=\beta_{k+1}^{2}\left[\left(g_{k+1}, g_{k+1}\right)-\sum_{l=1}^{k} \gamma_{k+1, l}^{2}\right]
$$

The methodology is well known in the literature for different geometric shapes of pupils (Swantner \& Chow 1994; Upton \& Ellerbroek 2004; Mahajan \& Dai 2007).

Still, the procedure establishes different basis sets depending on the order in which the seeds $g$ are fed into the procedure, and depending on which functional spaces they span.

\subsection{Zernike seeds}

To define a unique set $\left\{f_{j}\right\}$ of functions orthogonal over the binocular pupil, we may take the real and imaginary parts of the primitives (9) as the seeds in increasing order of complexity, i.e., increasing order of aberration and increasing $n$ and $m$. The Zernike polynomials $Z_{j}(z, \theta)$ over the full super-pupil of radius $R$ (normalized to $z=1$ ) in the Noll order of indexing (Noll 1976) are subduced into the two sub-apertures. There is an outer loop over $n=0,1,2, \ldots$ and an inner loop over $m=n \quad(\bmod 2), \ldots n$, considering only even $n-m$ :

$$
\begin{array}{r}
g_{1}=1 ; \quad g_{2}=z \cos \theta ; \quad g_{3}=z \sin \theta \\
g_{4}=2 z^{2}-1 ; \quad g_{5}=z^{2} \sin (2 \theta) ; \quad g_{6}=z^{2} \cos (2 \theta) \\
g_{7}=\left(3 z^{3}-2 z\right) \sin \theta ; \quad g_{8}=\left(3 z^{3}-2 z\right) \cos \theta ; \quad g_{9}=z^{3} \sin (3 \theta) ; \quad \ldots
\end{array}
$$

For this choice, the $n+1$ basis functions from $g_{1+n(n+1) / 2}$ up to and including $g_{(n+1)(n+2) / 2}$ are associated with a polynomial of order $n$ in $z$. The arithmetic 
remains real-valued, because the "atoms" of the seeds are the separated real and imaginary part of (9). (16) is split into

$$
\begin{aligned}
& \iint z^{n_{k}} \cos \left(m_{k} \theta\right) z^{n_{l}} \cos \left(m_{l} \theta\right) d \Omega=\frac{1}{2} A_{n_{k}+n_{l}, m_{k}+m_{l}}+\frac{1}{2} A_{n_{k}+n_{l}, \mid m_{k}-m_{l}}(24) \\
& \iint z^{n_{k}} \sin \left(m_{k} \theta\right) z^{n_{l}} \sin \left(m_{l} \theta\right) d \Omega=\frac{1}{2} A_{n_{k}+n_{l},\left|m_{k}-m_{l}\right|}-\frac{1}{2} A_{n_{k}+n_{l}, m_{k}+m_{l}} \\
& \iint z^{n_{k}} \cos \left(m_{k} \theta\right) z^{n_{l}} \sin \left(m_{l} \theta\right) d \Omega=0
\end{aligned}
$$

The first basis functions created with this recipe are discussed shortly in analytical form. We start with the global common piston $g_{1}$ which just needs to be normalized:

$$
f_{1}=\frac{1}{q} \frac{1}{\sqrt{2 \pi}}
$$

Next we feed what represents most of the differential piston, $g_{2}$, which turns out to be already orthogonal to $f_{1}$ and only needs to be normalized,

$$
f_{2}=\frac{1}{q} \sqrt{\frac{2}{\pi\left(4+q^{2}\right)}} z \cos \theta .
$$

Next we feed $g_{3}$, some common sideways tilt perpendicular to the baseline between the two pupils,

$$
f_{3}=\frac{1}{q^{2}} \sqrt{\frac{2}{\pi}} z \sin \theta .
$$

The first case of nonzero overlap with an earlier basis function occurs when we use $g_{4}$ as a seed (some nodding tilt between the sub-pupils along the baseline), which has a nonzero component along $f_{1}$ :

$$
f_{4}=\frac{1}{q^{2}} \sqrt{\frac{6}{\pi\left(12+q^{2}\right)}}\left(z^{2}-\frac{2+q^{2}}{2}\right) .
$$

Feeding $g_{5}$ to $g_{8}$ we get

$$
\begin{gathered}
f_{5}=\frac{1}{q^{2}} \sqrt{\frac{3}{\pi\left(6+q^{2}\right)}} z^{2} \sin (2 \theta) . \\
f_{6}=\frac{1}{q} \sqrt{\frac{3}{\pi\left(18+q^{2}\right)\left(12+q^{2}\right)}}\left[\frac{12+q^{2}}{q^{2}} z^{2} \cos (2 \theta)-\frac{12}{q^{2}} z^{2}+5\right] . \\
f_{7}=\frac{2}{q^{3}} \sqrt{\frac{1}{\pi\left(12+q^{2}\right)}}\left[3 z^{3} \sin \theta-\left(3+2 q^{2}\right) z \sin \theta\right] . \\
f_{8}=\frac{2}{q^{2}} \sqrt{\frac{1}{\pi Q_{8}\left(4+q^{2}\right)}\left[3\left(4+q^{2}\right) z^{3} \cos \theta-\left(12+21 q^{2}+2 q^{4}\right) z \cos \theta\right] .}
\end{gathered}
$$

The abbreviation $Q_{8} \equiv 288+48 q^{2}+48 q^{4}+q^{6}$ is used.

Figures 2 and 3 illustrate the first of these functions for $q=1 / 2$. 

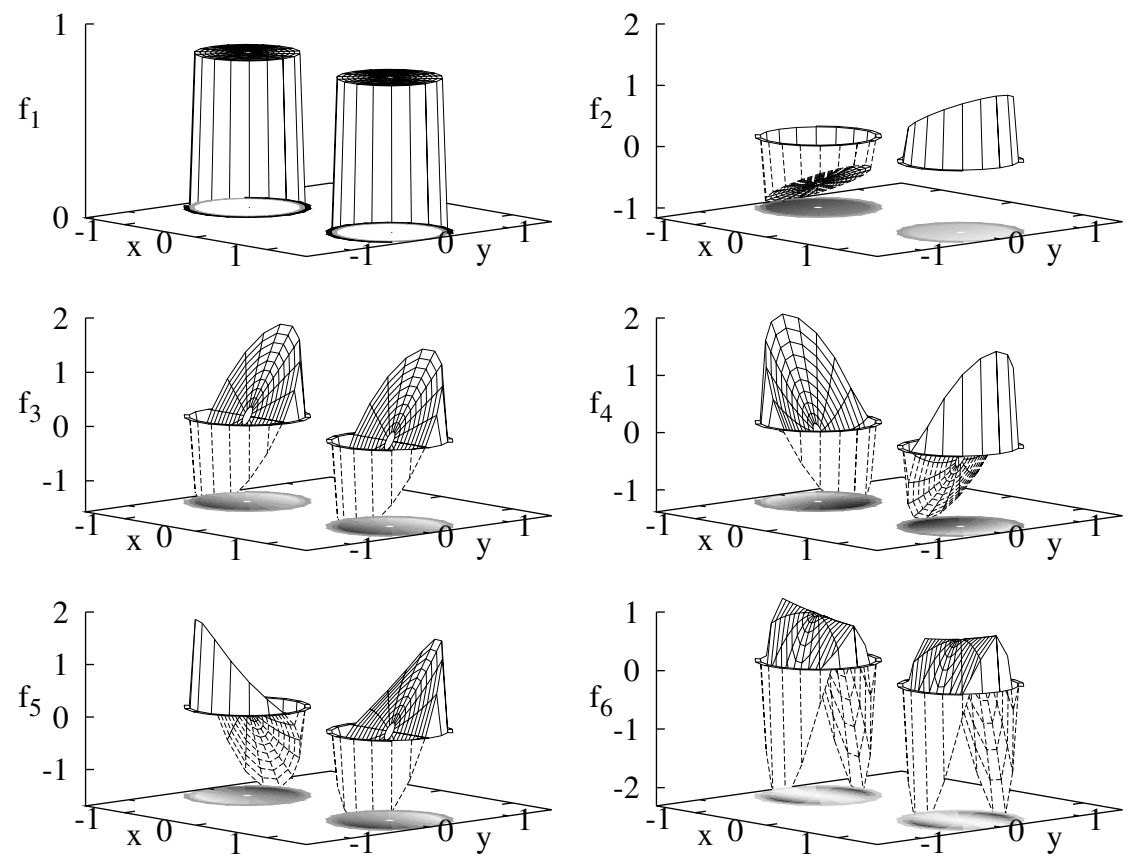

Fig. 2. The basis functions $f_{1}$ to $f_{6}$ for $q=1 / 2$.

Variants of this scheme exist. One may for example start from the Zernike functions defined in the two sub-pupils as the seeds and orthogonalize these. The choice above is steered towards cases where an external perturbation correlates beams; the vision is that spatial filtering this information rather than extrapolation of individual pupil information to the entire area is the task at hand.

\section{FOURIER REPRESENTATION}

All basis functions $f_{j}$ are linear superpositions of the primitive type (9) since $\cos (m \theta)=[\exp (i m \theta)+\exp (-i m \theta)] / 2$ and $\sin (m \theta)=[\exp (i m \theta)-\exp (-i m \theta)] /(2 i)$. The two-dimensional Fourier Transform of these is

$$
A_{n, m}(q, \boldsymbol{\sigma}) \equiv \int \exp (2 \pi i \boldsymbol{\sigma} \cdot \boldsymbol{z}) p_{n, m} d \Omega=\int \exp (2 \pi i \boldsymbol{\sigma} \cdot \boldsymbol{z}) z^{n} \exp (i m \theta) d \Omega
$$

for some wave number $\boldsymbol{\sigma}$. The limit $\sigma=0$ recovers $A_{n, m}(q, \boldsymbol{\sigma}=0)=A_{n, m}(q)$ in (10) as a special case. These integrals are decomposed by multipole expansions around the mid-points of the two sub-pupils that define local circular coordinates $s$ and $\varphi$ as in Figure 1 (Campbell 2003; Comastri et al. 2007; Lundström \& Unsbo 2007; Schwiegerling 2002; Shu et al. 2006): 

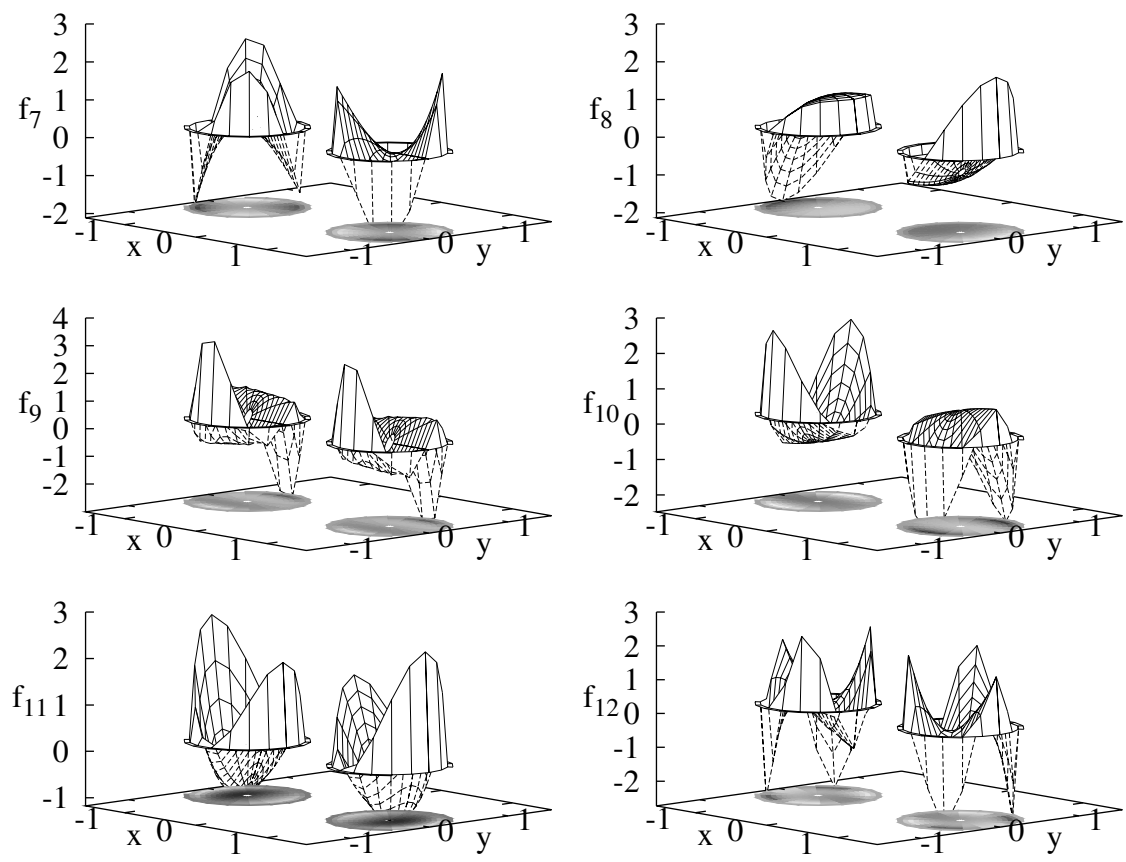

Fig. 3. The basis functions $f_{7}$ to $f_{12}$ for $q=1 / 2$.

$$
\begin{array}{r}
x=P+s \cos \varphi ; \quad y=s \sin \varphi \\
z^{2}=x^{2}+y^{2}=1+2 P s \cos \varphi+s^{2} ; \quad \theta=\arctan (y / x) \\
A_{n, m}(q, \boldsymbol{\sigma})=\sum_{P= \pm 1} e^{2 \pi i P \sigma_{x}} \int_{s \leq q} e^{2 \pi i \boldsymbol{\sigma} \cdot \boldsymbol{s}} z^{n} e^{i m \theta} d^{2} s
\end{array}
$$

where $\sigma_{x}$ is the component of $\sigma$ parallel to the baseline. Only the cases of even $n-m$ are of interest if we adhere to the basis of Section 4.2.

$$
\begin{aligned}
z^{n} e^{i m \theta} & =z^{n-m}\left(z e^{i \theta}\right)^{m}=\left(x^{2}+y^{2}\right)^{(n-m) / 2}(x+i y)^{m} \\
& =\left(1+2 P s \cos \varphi+s^{2}\right)^{(n-m) / 2}\left(P+s e^{i \varphi}\right)^{m}
\end{aligned}
$$

is expanded in multinomials of $x$ and $y$ and expresses $z^{n} e^{i m \theta}$ in the circular coordinates centered at $x=P$ with radial coordinate $s$ and azimuth $\varphi$. Powers of $P$ are reduced via $P^{2}=1$. Table 2 demonstrates cases for small $n$.

At this stage one could selectively drop terms of the form $s \exp ( \pm i \varphi), s \cos \varphi$ and $s \sin \varphi$ to investigate systems with individual tip-tilt compensation in the two sub-pupils.

This reduces each $A_{n, m}(q, \boldsymbol{\sigma})$ to a finite sum of integrals over circles of radius $q$ of the kind

$$
\int_{s \leq q} e^{2 \pi i \sigma \cdot s} s^{n} e^{i m \varphi} d^{2} s=e^{i m \varphi_{\sigma}} \int_{0}^{q} s^{n+1} d s \int_{0}^{2 \pi} e^{2 \pi i \sigma s \cos \varphi} e^{i m \varphi} d \varphi
$$


Table 2. Examples of the primitive basis function in the integral (38) translated to $(s, \varphi)$ coordinates. The cases of negative $m$ follow immediately by complexconjugation.

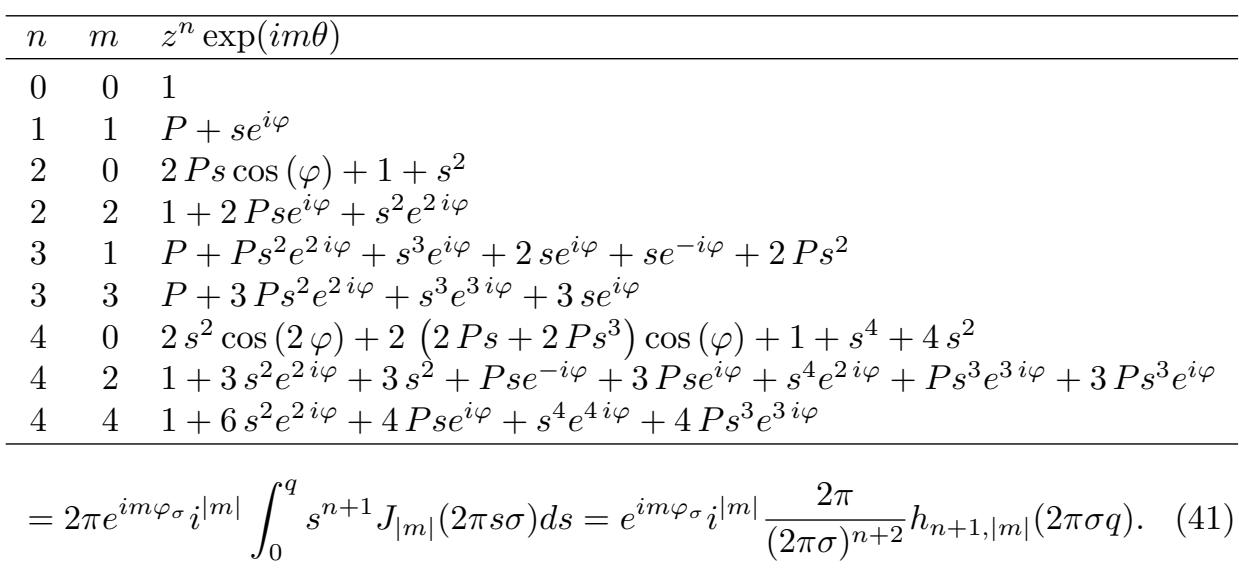

The azimuth $\varphi_{\sigma}$ of the wave number is defined with $\tan \varphi_{\sigma} \equiv \sigma_{y} / \sigma_{x}$. The integrals $h$ over Bessel functions $J$ are recursively accessible (Abramowitz \& Stegun 1972, 11.3.4) because $n-m$ is even:

$$
\begin{aligned}
\frac{1}{\alpha^{n+2}} h_{n+1, m}(\alpha) & \equiv \frac{1}{\alpha^{n+2}} \int_{0}^{\alpha} t^{n+1} J_{m}(t) d t \\
& =\frac{1}{\alpha} \sum_{j=0}^{(n-m) / 2}\left(\frac{m-n}{2}\right)_{j}\left(\frac{2}{\alpha}\right)^{j} J_{m+1+j}(\alpha) .
\end{aligned}
$$

We employ Pochhammer's symbol in this equation, defined as (Abramowitz \& Stegun 1972, 6.1.22)

$$
(x)_{j} \equiv x(x+1)(x+2) \cdots(x+j-1), j>0 ; \quad(x)_{0}=1 .
$$

The variable

$$
\alpha \equiv 2 \pi q \sigma
$$

takes the role of scale of resolution of the single telescope. The ideal plane-wave illumination parallel to the optical axis is represented by

$$
A_{0,0}(q, \boldsymbol{\sigma})=2 \cos \left(2 \pi \sigma_{x}\right) \frac{2 \pi q^{2}}{\alpha} J_{1}(\alpha)
$$

In the limit $\sigma=0,(40)$ simplifies to $\int_{s \leq q} s^{n} \exp (i m \varphi) d^{2} s=2 \pi \delta_{m, 0} q^{n+2} /(n+$ 2 ). We visualize the Fourier representations $\hat{f}_{j}(\sigma)$ of the first basis functions in Figure 4 . The $f_{j}(x, y)$ have a well defined parity with respect to reflection across the origin, i.e., the terms in (27)-(34) are superpositions of $z^{n} \sin (m \theta)$ and $z^{n} \cos (m \theta)$ with a fixed, common parity $(-1)^{m}$ in each line. As a consequence, the $\hat{f}_{j}\left(\sigma_{x}, \sigma_{y}\right)$ are purely real or purely imaginary, and only the non-vanishing of the two components is shown. 

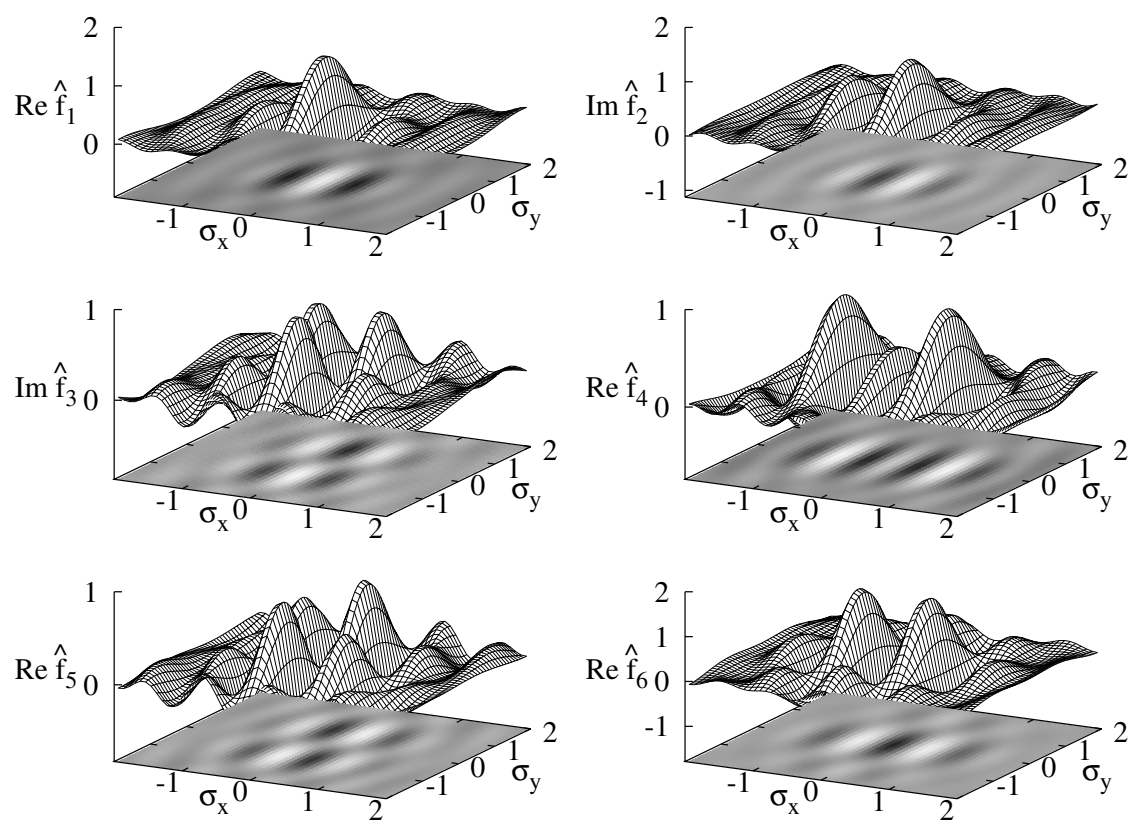

Fig. 4. The Fourier transforms of basis functions $f_{1}$ to $f_{6}$ for $q=1 / 2$.

$f_{1}(z, \theta)$ in Figure 2 is the convolution of the double pinhole mask (Santarsiero et al. 2007) with a single circular telescope pupil of area $\pi q^{2}$. Its Fourier representation $\hat{f}_{1}(\sigma)$ in Figure 4 is the familiar diffraction limited point spread function of the two-beam interferometer (Sabatke et al. 2006), the product of the circular Airy disk centered at $\sigma=0$ by the pattern of fringes with their narrower width along $\sigma_{x}$ as set by the baseline length:

$$
\hat{f}_{1}(\boldsymbol{\sigma})=\sqrt{\frac{2}{\pi}} \cos \left(2 \pi \sigma_{x}\right) \frac{J_{1}(2 \pi \sigma q)}{\sigma} .
$$

$\hat{f}_{2}$ and $\hat{f}_{3}$ are purely imaginary:

$$
\begin{gathered}
\hat{f}_{2}(\boldsymbol{\sigma})=\sqrt{\frac{2}{4+q^{2}}} \frac{2 i}{\sigma \pi^{1 / 2}}\left[q \cos \varphi_{\sigma} \cos \left(2 \pi \sigma_{x}\right) J_{2}(2 \pi \sigma q)+\sin \left(2 \pi \sigma_{x}\right) J_{1}(2 \pi \sigma q)\right], \\
\hat{f}_{3}(\boldsymbol{\sigma})=\frac{2^{3 / 2} i \cos \left(2 \pi \sigma_{x}\right) \sin \varphi_{\sigma}}{\pi^{1 / 2} \sigma} J_{2}(2 \pi \sigma q) .
\end{gathered}
$$

In the standard application, $f_{1}$ is the ideal, unperturbed field of illumination along the optical axis, and the higher basis functions carry the information on the (Seidel) aberrations. Amplitude and phase are conveniently encoded in complex numbers. Expansion of the phasors up to linear order shows that for small perturbations the $f_{\geq 2}$ can be considered purely imaginary numbers, because the real parts can be absorbed in $f_{1}$. (Phases are in that sense more important than amplitude variations in astronomical interferometry.) Each pupil-plane component 
$f_{j}$ turns into a unique contribution $\hat{f}_{j}$ in the image plane, because the Fourier Transform is linear. The detector records the intensity, the absolute square of the sum of the amplitudes.

This text book summary remains correct for $f_{2}$ and $f_{3}$ : the imaginary units implied by the expansion of the phasors and created by the Fourier Transform basically annihilate; the $\hat{f}_{2,3}$ contributions can be linearly added on top of $\hat{f}_{1}$. Their effect on $\hat{f}_{1}$ in an intensity (contemporary optical or infrared) interferometer is to shift the center fringe, as expected from Figure 2 , since $f_{2}$ and $f_{3}$ represent plane waves from off-center targets. The patterns of $\hat{f}_{4}-\hat{f}_{6}$ do not experience this product of imaginary units; their phasors remain orthogonal to $\hat{f}_{1}$ in the complex plane, so their contribution are only of second order in the intensity. They mainly enhance the two side-lobes relative to the center fringe. In practice, unless the setup is part of a seeing monitor, $\hat{f}_{6}$ will be the first in this sequence basically unaffected by tip-tilt systems monitoring both sub-pupils.

\section{SPECKLE INTERFEROMETRY}

\subsection{Modal decomposition}

The conceptional advantage of the Gram-Schmidt orthogonalization in the first chapters is that the basis functions are never more complicated than admitted by the seeds. The output, however, remains anonymous if the functions expanded in that basis are not associated with some physical field.

A different approach towards orthogonal basis functions are eigenvectors of symmetric (hermitian) linear binary operators. Modeling of stochastic atmospheric turbulence is the case example that shall be worked out in this section. The field is the phase of the electromagnetic wave as a function of the pupil plane coordinate.

The following lines write down the core equations so all variables are defined. The sole new aspect beyond the well established theory (Fried 1978) is the shape of the pupil mask. The spectral density for Kolmogorov turbulence with exponent $\gamma=2 / 3$, Fried radius $r_{0}$, and spatial frequency $f$ is (Fried 1966; Roddier 1981; Mathar 2010)

$$
C_{\varphi}(f)=-c_{\varphi} \frac{\Gamma\left(\frac{3+\gamma}{2}\right)}{\pi^{2+\gamma} \Gamma\left(-\frac{1+\gamma}{2}\right)} \frac{1}{r_{0}^{1+\gamma}} f^{-3-\gamma} \approx 0.0228955 \frac{1}{r_{0}^{5 / 3}} \frac{1}{f^{11 / 3}} .
$$

A constant $c_{\varphi}$ appears while the phase structure constant is molded into $r_{0}$,

$$
2 c_{\varphi}=2\left[4 \Gamma\left(\frac{3+\gamma}{1+\gamma}\right)\right]^{(1+\gamma) / 2} \approx 6.883877
$$

The Karhunen-Loève eigenvalue equation which diagonalizes the covariance matrix in terms of orthogonal eigenfunctions $\mathcal{K}_{j}$ is

$$
\int d \Omega_{R}^{\prime} \int d^{2} f C_{\varphi}(f) e^{-2 \pi \boldsymbol{f} \cdot\left(\boldsymbol{r}-\boldsymbol{r}^{\prime}\right)} \mathcal{K}_{j}\left(r^{\prime}\right)=\mathcal{B}_{j}^{2} \mathcal{K}_{j}(r)
$$

In association with normalized $\mathcal{K}, \mathcal{B}$ are the strengths (weights) in the vector of randomized expansion coefficients for each phase screen instance. 
Scaling length coordinates by $R$ as in (5) and spatial frequencies as $f=\sigma / R$ yields

$$
-c_{\varphi} \frac{\Gamma\left(\frac{3+\gamma}{2}\right)}{2(2 \pi)^{2+\gamma} \Gamma\left(-\frac{1+\gamma}{2}\right)} \int d \Omega^{\prime} \int d^{2} \sigma \frac{1}{\sigma^{3+\gamma}} e^{-2 \pi \boldsymbol{\sigma} \cdot\left(\boldsymbol{x}-\boldsymbol{x}^{\prime}\right)} K_{j}\left(\boldsymbol{x}^{\prime}\right)=\lambda_{j}^{2} K_{j}(\boldsymbol{x})
$$

with unit-less eigenvalues and eigenvectors

$$
\lambda_{j}^{2} \equiv \frac{\mathcal{B}_{j}}{(2 R)^{2}}\left(\frac{r_{0}}{2 R}\right)^{1+\gamma}, \quad K_{j}(\boldsymbol{x})=R \mathcal{K}(\boldsymbol{r}),
$$

and the tighter format

$$
-c_{\varphi} \frac{\Gamma\left(\frac{3+\gamma}{2}\right)}{2(2 \pi)^{2+\gamma} \Gamma\left(-\frac{1+\gamma}{2}\right)} \frac{1}{\sigma^{3+\gamma}} K_{j}(\boldsymbol{\sigma})=\lambda_{j}^{2} K_{j}(\boldsymbol{\sigma}) .
$$

One might equally scale variables not with $R$ but with $r$. The choice depends on whether the interferometer is considered a masked variant of a really large telescope or rather two single telescopes with some coherence properties.

To advance to a standard numerical task of linear algebra, one may expand $K$ in the piston-depleted primitive basis

$$
K_{j}(\boldsymbol{x})=\sum_{n, m} \kappa_{n, m, j}\left(z^{n} e^{i m \theta}-\frac{1}{2 \pi q^{2}} A_{n, m}(q)\right) .
$$

Moving on to Fourier space, the primitive basis and expansion become

$$
\begin{gathered}
\bar{A}_{n, m}(q, \boldsymbol{\sigma}) \equiv A_{n, m}(q, \boldsymbol{\sigma})-\frac{1}{2 \pi q^{2}} A_{n, m}(q) A_{0,0}(q, \boldsymbol{\sigma}), \\
K_{j}(\boldsymbol{\sigma})=\sum_{n, m} \kappa_{n, m, j} \bar{A}_{n, m}(q, \boldsymbol{\sigma})
\end{gathered}
$$

which converts (54) to a generalized eigenvalue equation for the unknown vector of coefficients $\kappa$ of mode $j$ :

$$
\sum_{n, m} I_{n^{\prime \prime}, m^{\prime \prime}, n, m} \kappa_{n, m, j}=\lambda_{j}^{2} \sum_{n^{\prime}, m^{\prime}} O_{n^{\prime \prime}, m^{\prime \prime}, n^{\prime}, m^{\prime}} \kappa_{n^{\prime}, m^{\prime}, j}
$$

Its overlap matrix elements $O$ are easily evaluated in real space

$$
\begin{aligned}
O_{n^{\prime \prime}, m^{\prime \prime}, n^{\prime}, m^{\prime}} & \equiv \int d^{2} \sigma \bar{A}_{n^{\prime \prime}, m^{\prime \prime}}^{*}(q, \boldsymbol{\sigma}) \bar{A}_{n^{\prime}, m^{\prime}}(q, \boldsymbol{\sigma}) \\
& =A_{n^{\prime \prime}+n^{\prime}, m^{\prime}-m^{\prime \prime}}(q)-\frac{1}{2 \pi q^{2}} A_{n^{\prime}, m^{\prime}}(q) A_{n^{\prime \prime}, m^{\prime \prime}}(q) .
\end{aligned}
$$

A technique to evaluate its correlator matrix elements

$$
I_{n^{\prime \prime}, m^{\prime \prime}, n, m} \equiv-c_{\varphi} \frac{\Gamma\left(\frac{3+\gamma}{2}\right)}{2(2 \pi)^{2+\gamma} \Gamma\left(-\frac{1+\gamma}{2}\right)} \int d^{2} \sigma \frac{1}{\sigma^{3+\gamma}} \bar{A}_{n^{\prime \prime}, m^{\prime \prime}}^{*}(q, \boldsymbol{\sigma}) \bar{A}_{n, m}(q, \boldsymbol{\sigma})
$$

is outlined in Appendix C. Figures 5 and 6 show the 12 strongest modes $K_{j}(\mathbf{x})$, sorted along decreasing $\lambda_{j}^{2}$ for the same $q$ as above. 

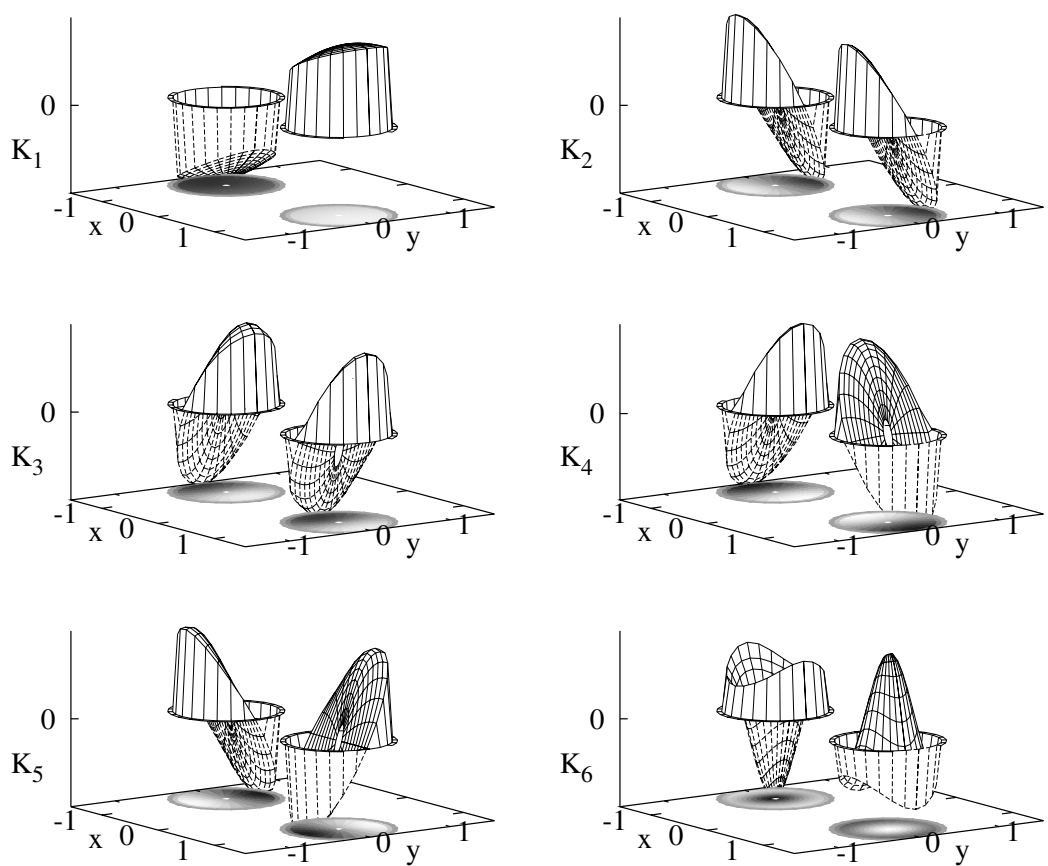

Fig. 5. The basis functions $K_{1}$ to $K_{6}$ for $q=1 / 2 . \lambda_{j}^{2}$ is $0.14273,0.01810$, $0.01659,0.01372,0.01286$ and 0.00390 for $j=1$ to 6 . The total power is estimated to $\sum_{j=1}^{90} \lambda_{j}^{2}=0.2585$.

\subsection{Symmetries}

In overview, we recognize a compositional principle: the familiar modes of the single circular pupil are placed at one sub-aperture, and copies with even and odd parity relative to the baseline center at the other. The quadruplet $\left[K_{7}, K_{8}\right.$, $K_{9}, K_{11}$ ] originates from two bi-polar modes of the circular pupil, for example. A similar quadruplet is $\left[K_{12}, K_{13}, K_{16}, K_{17}\right]$ from three tri-polar modes. The cross-correlation induces additional deformations, demonstrated for example by the arched rims of $K_{6}$ and $K_{10}$.

The functions are classified with the quadrilateral symmetry of the stripe pupil (Mathar 2010). Solving (60) emits these functions with correct symmetry and multiplicity. The qualitative implication for on-axis interferometric recombination, which adds field amplitudes that share the same $(s, \varphi)$ coordinates in the two sub-apertures, is: components like $K_{2}, K_{3}$ and $K_{9}-K_{12}$ which display the same phase at the associated points add fully constructively/destructively in the two interferometric outputs generated by the beam combiner. Components like $K_{4}-K_{8}$ with opposite phases contribute to the visibility loss because their superpositions remain imperfect and their intensity is channeled (split) into both outputs. If the optics is based on off-axis (imaging) recombination, the impact of symmetry on the Fourier transforms of the modes $K$ is equivalent to the impact on the Fourier transforms of the $f$-bases in Section 5: shifting, strengthening/weakening of the center and side lobes. 

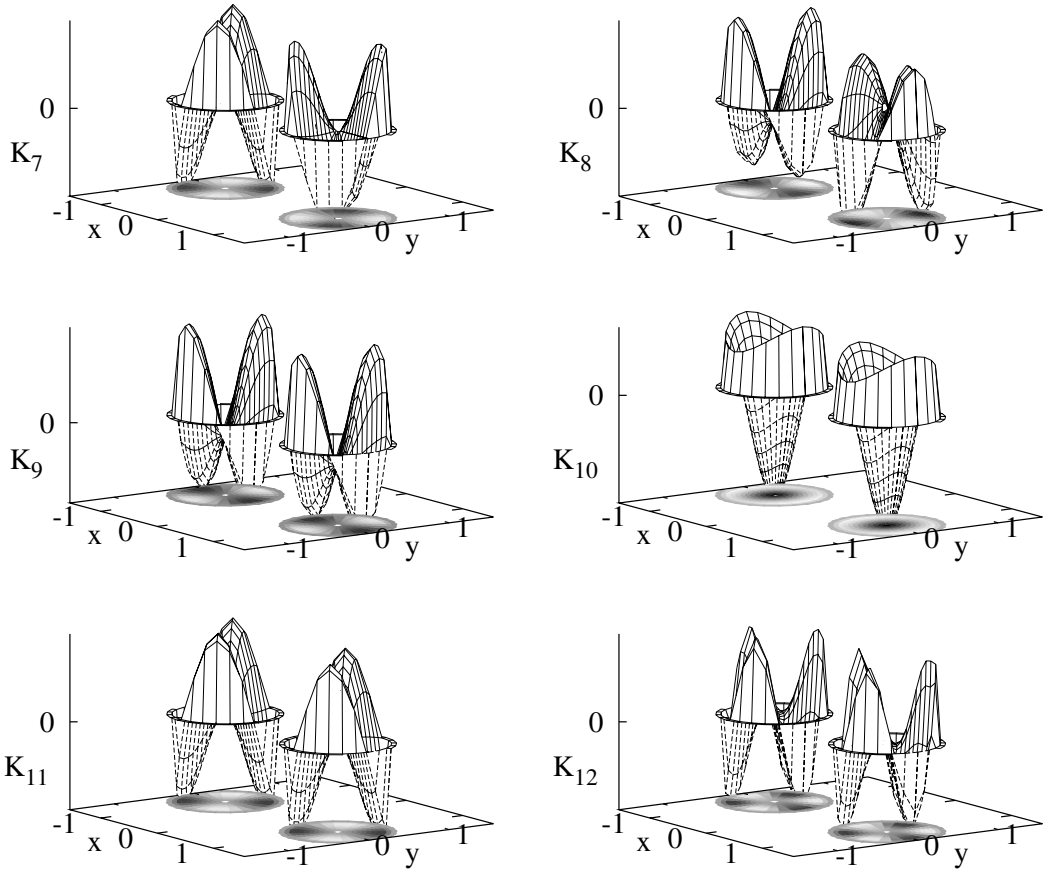

Fig. 6. The basis functions $K_{7}$ to $K_{12}$ for $q=1 / 2 . \lambda_{j}^{2}$ is $0.00390,0.00383$, $0.00377,0.00376,0.00340$ and 0.00168 for $j=7$ to 12 .

This extends results known in the limit of long baselines, $q \rightarrow 0$, (Roddier \& Roddier 1976) to finite baselines. The eigenvalues $\lambda^{2}$ introduce quantitative aspects that are absent in Section 4: A fringe tracker is essentially sensitive to a component of $K_{1}$, whereas the tip-tilt stages are experiencing the power spectra of modes $K_{1}$ to $K_{5}$. Building ratios of power density functions among these offers one route to measure outer scales of turbulence; within the Kolmogorov model, the relative importance is $\lambda_{1}^{2} / \lambda_{2}^{2} \propto 1 / q^{3+\gamma}$ as $q \rightarrow 0$.

\section{SUMMARY}

Orthogonality of functions over the area of two non-overlapping circles is defined according to standard algebra. The fundamental areal integral for functions that are of simple analytical format in the circular coordinate system attached to the center of symmetry has been recursively reduced to polynomials or Gaussian Hypergeometric Functions of the normalized circle radius. This defines a set of orthogonal basis functions over the common area, if the Zernike basis functions, for example, provide the input to the Gram-Schmidt procedure. The complete information for numerical instantiation has been presented.

There is a common mathematical ground with the modal decomposition of Kolmogorov phase screens if the same simple functions are used to span the eigenvector space of the Karhunen-Loève equation. The hypergeometric expansions of the key integrals are available in the literature. 


\section{APPENDIX A. AUXILIARY INTEGRAL $B$}

This section evaluates of the integrals defined in (14). A square of the $(n+2)$ nd power is split off and expanded,

$$
B_{n+2, m}^{ \pm}(q)=\left(q^{2}-1\right) B_{n, m}^{ \pm}(q)+2 X_{n+1, m}^{ \pm}(q),
$$

where we have introduced

$$
X_{n+1, m}^{ \pm}(q) \equiv \int_{0}^{\arcsin q} \cos \theta\left[\cos \theta \pm \sqrt{q^{2}-\sin ^{2} \theta}\right]^{n+1} \cos (m \theta) d \theta .
$$

This defines a first strategy to evaluate $B_{n+2, m}(q)$ : recursive reduction of the first lower index in steps of two at the expense of implementing the $X_{n+1, m}(q)$ integrals - to be treated in Appendix B. A recursion starting from an even $n$ ends up at

$$
B_{0, m}^{ \pm}(q)=\frac{1}{m} \sin (m \arcsin q) \simeq 0 .
$$

This is also correct in the limit $m \rightarrow 0$, and equivalent to zero as the difference $B_{0, m}^{+}-B_{0, m}^{-}$is formed. The symbol $\simeq$ indicates in this script that terms have been omitted on the right hand side which cancel in differences $B^{+}(q)-B^{-}(q)$ (common pair of subscripts with both $B$ ) or in differences $X^{+}(q)-X^{-}(q)$ (common pair of subscripts with both $X)$. For odd $n$, the recursion (61) terminates at

$$
\begin{aligned}
B_{1, m}^{ \pm}(q) & =\int_{0}^{\arcsin q} d \theta\left(\cos \theta \pm \sqrt{q^{2}-\sin ^{2} \theta}\right) \cos (m \theta) \\
& \simeq \pm \int_{0}^{\arcsin q} d \theta \sqrt{q^{2}-\sin ^{2} \theta} T_{m}(\cos \theta) .
\end{aligned}
$$

For larger $m$, a mixture of partial integrations and the product rule for Chebyshev polynomials $T$ (Abramowitz \& Stegun 1972, 22.7.24) generates a recursion for the second index in steps of two (Novario 2005):

$$
B_{1, m}^{ \pm}(q) \simeq \frac{2(m-2)\left(1-2 q^{2}\right)}{m+1} B_{1, m-2}^{ \pm}(q)+\frac{5-m}{m+1} B_{1, m-4}^{ \pm}(q) .
$$

Starting values of this recursion at small even $m$ are elliptic integrals and are not discussed here - they imply that $n+m$ is odd which does not happen for the seeds proposed in Section 4 or the basis in Section 6. At small odd $m$, the recursion starts from

$$
B_{1,1}^{ \pm}(q)= \pm \frac{\pi}{4} q^{2} ; \quad B_{1,3}^{ \pm}(q)= \pm \frac{\pi}{4} q^{2}\left(1-q^{2}\right) .
$$

An alternative second strategy to evaluate $B_{n+2, m}(q)$ looks as follows: Binomial expansion of the $(n+2)$ nd power in the integrand of (14) yields

$$
\begin{aligned}
B_{n+2, m}^{ \pm}(q) \simeq \quad & \pm \sum_{s=0}^{\lfloor(n+1) / 2\rfloor}\left(\begin{array}{c}
n-s+1 \\
s
\end{array}\right)\left(q^{2}-1\right)^{s} \\
& \times \sum_{u=0, n-u \text { odd }}^{n+1-2 s}\left(\begin{array}{c}
n+1-2 s \\
\frac{n+1-u}{2}-s
\end{array}\right)\left[B_{1, u+m}^{ \pm}(q)+B_{1,|u-m|}^{ \pm}(q)\right] .
\end{aligned}
$$


The prime at the sum symbol means the term for $u=0$ is to be halved. The decrement of the first index of $B_{n+2, m}^{ \pm}$via (68) down to 1 comes at the cost of an increment of some second indices, eventually a recourse to (66). Examples of (68) for small $n$ are:

$$
\begin{gathered}
B_{2, m}^{ \pm} \simeq B_{1, m+1}+B_{1, m-1}, \quad m \geq 2 \\
B_{3, m}^{ \pm} \simeq B_{1, m+2}+B_{1, m-2}+\left(1+q^{2}\right) B_{1, m}, \quad m \geq 2 \\
B_{4,2}^{ \pm} \simeq 2\left(1+q^{2}\right) B_{1,1}+\left(1+2 q^{2}\right) B_{1,3}+B_{1,5} \\
B_{4, m}^{ \pm} \simeq B_{1, m-3}+\left(1+2 q^{2}\right) B_{1, m-1}+\left(1+2 q^{2}\right) B_{1, m+1}+B_{1, m+3}, \quad m \geq 3 \\
B_{5,2}^{ \pm} \simeq\left(1+3 q^{2}\right) B_{1,0}+\left(2+4 q^{2}+q^{4}\right) B_{1,2}+\left(1+3 q^{2}\right) B_{1,4}+B_{1,6} \\
B_{5,3}^{ \pm} \simeq\left(2+3 q^{2}\right) B_{1,1}+\left(1+4 q^{2}+q^{4}\right) B_{1,3}+\left(1+3 q^{2}\right) B_{1,5}+B_{1,7}
\end{gathered}
$$

The argument $q$ at the $B_{., .}(q)$ has been omitted for brevity.

\section{APPENDIX B. AUXILIARY INTEGRAL $X$}

The auxiliary integrals (62) are put into an algebraic format by the substitution $\sin \theta \equiv q z$

$$
X_{n+1, m}^{ \pm}(q)=q \int_{0}^{1}\left(\sqrt{1-q^{2} z^{2}} \pm q \sqrt{1-z^{2}}\right)^{n+1} T_{m}\left(\sqrt{1-q^{2} z^{2}}\right) d z
$$

and then broken down through binomial expansion of the $(n+1)$ st power and the explicit polynomial representation of the Chebyshev function (Abramowitz \& Stegun 1972, 22.3.6) (Gradstein \& Ryshik 1981 3.197.3)

$$
\begin{aligned}
& X_{n+1, m}^{ \pm}(q) \simeq \pm 2^{m-1} \pi(n+1) ! \sum_{j=0}^{\lfloor n / 2\rfloor} \frac{1}{(n-2 j) ! j !}\left(\frac{q}{2}\right)^{2 j+2} \\
& \times \begin{cases}\left.\sum_{\sigma=0}^{\lfloor m / 2\rfloor}\left(-\frac{1}{4}\right)^{\sigma} \frac{m}{2(m-\sigma)}\left(\begin{array}{c}
m-\sigma \\
\sigma
\end{array}\right){ }_{2} F_{1}\left(\begin{array}{c}
j+\sigma-\frac{n+m}{2}, \frac{1}{2} \\
j+2
\end{array}\right) ; q^{2}\right) ; & m>0 ; \\
{ }_{2} F_{1}\left(\begin{array}{c}
j-\frac{n}{2}, \frac{1}{2} \\
j+2
\end{array} \mid q^{2}\right) ; & m=0,\end{cases}
\end{aligned}
$$

where the Gaussian Hypergeometric Series is (Abramowitz \& Stegun 1972, §15)

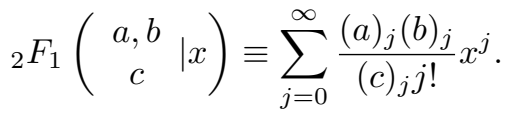

If $n+m$ is an even number - the case for the Gram-Schmidt procedure described in Section 4.2 - the hypergeometric series terminate and become polynomials of $q^{2}$ of order $(n+m) / 2-j-\sigma$.

\section{APPENDIX C. KOLMOGOROV COVARIANCE MATRIX ELEMENTS}

Combining (38) to (42), the ingredients of (60) are of the form

$$
\bar{A}_{n, m}(q, \boldsymbol{\sigma})=\sum_{n^{\prime}=0}^{n} \sum_{m^{\prime}=-n^{\prime}}^{n^{\prime}} \sum_{j \geq 0} \cdots e^{2 \pi i P \sigma \cos \varphi_{\sigma}} e^{i m^{\prime} \varphi_{\sigma}} \alpha^{-j-1} J_{m^{\prime}+1+j}(\alpha) .
$$


The dots indicate coefficients that do not depend on $\sigma$ and are in that respect immaterial to the subsequent analysis of the structure of the $d^{2} \sigma$-integrals.

We assume that the piston has been removed as requested by (56) in conjunction with (45): for all $m=0$, components proportional to $\frac{1}{\alpha} J_{1}(\alpha)$ are projected out by the recurrence of the Bessel functions (Abramowitz \& Stegun 1972, 9.1.27):

$$
\frac{1}{\alpha^{j+1}} J_{1+j}(\alpha)=\sum_{l=0}^{j-1} \frac{(j-l) !}{2^{l+1}(1+j) ! \alpha^{j-l}} J_{2+j-l}(\alpha)+\frac{1}{2^{j}(1+j) !} \frac{1}{\alpha} J_{1}(\alpha) .
$$

The net coefficient in front of the last term becomes zero, which eliminates the divergent cases of the integrals further down.

The matrix elements in (60) are essentially integrals over products of these,

$$
\begin{array}{r}
M \equiv \int d^{2} \sigma \frac{1}{\sigma^{3+\gamma}} e^{-2 \pi i P \sigma \cos \varphi_{\sigma}} e^{-i m \varphi_{\sigma}} \alpha^{-l-1} J_{m+1+j}(\alpha) \\
\times e^{2 \pi i P^{\prime} \sigma \cos \varphi_{\sigma}} e^{i m^{\prime} \varphi_{\sigma}} \alpha^{-l^{\prime}-1} J_{m^{\prime}+1+j^{\prime}}(\alpha) \\
=(2 \pi q)^{1+\gamma} \int_{0}^{\infty} d \alpha \int_{0}^{2 \pi} d \varphi_{\sigma} \frac{1}{\alpha^{3+\gamma+l+l^{\prime}}} e^{i\left[\left(P^{\prime}-P\right) / q\right] \alpha \cos \varphi_{\sigma}} e^{i\left(m^{\prime}-m\right) \varphi_{\sigma}} \\
\times J_{m+1+j}(\alpha) J_{m^{\prime}+1+j^{\prime}}(\alpha) .
\end{array}
$$

The integration over $\varphi_{\sigma}$ for integer $m^{\prime}-m$ follows the scheme

$$
\int_{0}^{2 \pi} e^{i v \cos \phi+i m \phi} d \phi=2 \pi i^{|m|} J_{|m|}(v)
$$

leading to a triple-product of Bessel functions

$$
\begin{gathered}
M=\quad 2 \pi i^{\left|m^{\prime}-m\right|}(2 \pi q)^{1+\gamma} \int_{0}^{\infty} d \alpha \frac{1}{\alpha^{3+\gamma+l+l^{\prime}}} \\
\times J_{\left|m^{\prime}-m\right|}\left[\left(P^{\prime}-P\right) \alpha / q\right] J_{m+1+j}(\alpha) J_{m^{\prime}+1+j^{\prime}}(\alpha) \\
= \begin{cases}0, & P^{\prime}=P, m^{\prime} \neq m ; \\
2 \pi(2 \pi q)^{1+\gamma} \int_{0}^{\infty} \frac{d \alpha}{\alpha^{3+\gamma+l+l^{\prime}} J_{m+1+j}(\alpha) J_{m^{\prime}+1+j^{\prime}}(\alpha),} & P^{\prime}=P, m^{\prime}=m ; \\
2 \pi i^{\left|m^{\prime}-m\right|}(2 \pi q)^{1+\gamma} \int_{0}^{\infty} \frac{d \alpha}{\alpha^{3+\gamma+l+l^{\prime}}} & \\
\quad \times J_{\left|m^{\prime}-m\right|}\left(2 P^{\prime} \alpha / q\right) J_{m+1+j}(\alpha) J_{m^{\prime}+1+j^{\prime}}(\alpha), & P^{\prime}=-P .\end{cases}
\end{gathered}
$$

The first and middle cases are familiar parity selection rules and integrals of a single circular pupil (Noll 1976). The third, the cross-pupil case is (Fabrikant 2003; Bailey 1936)

$$
\begin{array}{r}
\int_{0}^{\infty} t^{v-1} J_{\rho}(c t) J_{\mu}(t) J_{\nu}(t) d t=\frac{2^{v-1} \Gamma\left(\frac{v+\mu+\nu+\rho}{2}\right)}{c^{v+\mu+\nu} \Gamma(\mu+1) \Gamma(\nu+1) \Gamma\left(1-\frac{v+\mu+\nu-\rho}{2}\right)} \\
\times F_{4}\left(\frac{v+\mu+\nu-\rho}{2}, \frac{v+\mu+\nu+\rho}{2} ; \mu+1, \nu+1 ; \frac{1}{c^{2}}, \frac{1}{c^{2}}\right) .
\end{array}
$$


This involves the Appell function $F_{4}$

$$
F_{4}\left(a, b ; c ; c^{\prime} ; x, y\right) \equiv \sum_{m=0}^{\infty} \sum_{n=0}^{\infty} \frac{(a)_{m+n}(b)_{m+n}}{(c)_{m}\left(c^{\prime}\right)_{n} m ! n !} x^{m} y^{n}
$$

with contiguous relations (Appell \& Kampé de Fériet 1926)

$$
\begin{array}{r}
\frac{b}{c} x F_{4}\left(a+1, b+1 ; c+1 ; c^{\prime} ; x, y\right)+\frac{b}{c^{\prime}} y F_{4}\left(a+1, b+1 ; c ; c^{\prime}+1 ; x, y\right) \\
=F_{4}\left(a+1, b ; c ; c^{\prime} ; x, y\right)-F_{4}\left(a, b ; c ; c^{\prime} ; x, y\right)
\end{array}
$$

and

$$
a F_{4}\left(a+1, b ; c, c^{\prime} ; x, y\right)-b F_{4}\left(a, b+1 ; c, c^{\prime} ; x, y\right)=(a-b) F_{4}\left(a, b ; c, c^{\prime} ; x, y\right) .
$$

In our case with equal arguments it can be formulated as a generalized hypergeometric series (Slater 1966)

$$
{ }_{p} F_{q}\left(\begin{array}{c}
a_{1}, a_{2}, \ldots, a_{p} \\
b_{1}, b_{2}, \ldots, b_{q}
\end{array} \mid x\right) \equiv \sum_{m=0}^{\infty} \frac{\left(a_{1}\right)_{m}\left(a_{2}\right)_{m} \cdots\left(a_{p}\right)_{m}}{\left(b_{1}\right)_{m}\left(b_{2}\right)_{m} \cdots\left(b_{q}\right)_{m} m !} x^{m}
$$

as (Erdélyi et al. 1954, p. 350)

$$
F_{4}\left(a, b ; c ; c^{\prime} ; x, x\right)={ }_{4} F_{3}\left(\begin{array}{c}
a, b, \frac{c+c^{\prime}}{2}, \frac{c+c^{\prime}-1}{2} \\
c, c^{\prime}, c+c^{\prime}-1
\end{array} \mid 4 x\right)
$$

or by summation over products of Gauss Hypergeometric Functions (Burchnall \& Chaundy 1942)

$$
\begin{aligned}
F_{4}\left(a, b ; c, c^{\prime} ; x, x\right)= & \sum_{r \geq 0} \frac{(a)_{r}(b)_{r}\left(a+b-c-c^{\prime}+1\right)_{r}}{r !(c)_{r}\left(c^{\prime}\right)_{r}} t^{2 r} \\
& \times{ }_{2} F_{1}\left(\begin{array}{c}
a+r, b+r \\
c+r
\end{array} \mid t\right){ }_{2} F_{1}\left(\begin{array}{c}
a+r, b+r \\
c^{\prime}+r
\end{array} \mid t\right),
\end{aligned}
$$

where $t \equiv(1-\sqrt{1-4 x}) / 2$.

Outlook to an extension: there is also the prospect to study finite outer scales with a von-Kármán spectrum. The wavenumber dispersion in (81) is substituted as $1 / \alpha^{3+\gamma+l+l^{\prime}} \rightarrow 1 /\left[\alpha^{l+l^{\prime}}\left(\alpha^{2}+\alpha_{0}^{2}\right)^{(3+\gamma) / 2}\right]$, where $\alpha_{0}$ is defined by insertion of the cut-off wavenumber in (44). The middle line in (81) remains known territory then (Mathar 2009). The structure of $M$ in the third line is more challenging:

$$
2 \pi i^{\left|m^{\prime}-m^{\prime}\right|}(2 \pi q)^{1+\gamma} \int_{0}^{\infty} \frac{d \alpha}{\alpha^{l+l^{\prime}+3+\gamma}\left[1+\left(\alpha_{0} / \alpha\right)^{2}\right]^{(3+\gamma) / 2}} J_{\rho}(c \alpha) J_{\nu}(\alpha) J_{\mu}(\alpha) .
$$

The triple product is (Landau \& Luswili 2001)

$$
\begin{array}{r}
J_{\rho}(c z) J_{\nu}(z) J_{\mu}(z)=\frac{(c z / 2)^{\rho}(z / 2)^{\nu+\mu}}{\Gamma(\rho+1) \Gamma(\nu+1) \Gamma(\mu+1)}{ }_{0} F_{1}\left(\begin{array}{c}
- \\
\rho+1
\end{array} \mid-(c z / 2)^{2}\right) \\
\quad \times{ }_{2} F_{3}\left(\begin{array}{c}
1+\frac{\mu+\nu}{2}, \frac{1+\nu+\mu}{2} \\
\nu+1, \mu+1, \nu+\mu+1
\end{array} \mid-z^{2}\right) .
\end{array}
$$


As its Mellin transform is known (Miller 1997), the integral becomes a sum of two hypergeometric series (Takato \& Yamaguchi 1995).

\section{REFERENCES}

Abramowitz M., Stegun I. A., eds. 1972, Handbook of Mathematical Functions, Dover Publications, New York

Appell P., Kampé de Fériet J. 1926, Fonctions Hypergéométriques et Hypersphériques. Polynomes d'Hermite, Gauthier-Villars, Paris

Arguijo P., Scholl M. S. 2003, Appl. Opt., 42, 3284

Bailey W. N. 1936, Proc. Lond. Math. Soc., 40, 37

Bhatia A. B., Wolf E. 1952, Proc. Phys. Soc. B, 65, 909

Burchnall J. L., Chaundy T. W. 1942, Quart. J. Math., 11, 249

Campbell C. E. 2003, J. Opt. Soc. Am., A, 20, 209

Chong C.-W., Raveendran P., Mukundan R. 2003, Pattern Recognition, 36, 731

Comastri S. A., Perez L. I., Pérez G. D., Martin G., Bastida K. 2007, J. Opt. A: Pure and Appl. Opt., 9, 209

Dai G.-m., Mahajan V. N. 2007a, Opt. Lett., 32, 74

Dai G.-m., Mahajan V. N. 2007b, J. Opt. Soc. Am., A, 24, 139

Erdélyi A., Magnus W., Oberhettinger F., Tricomi F. G., eds. 1954, Tables of Integral Transforms, vol. 2, McGraw-Hill, New York,London

Fabrikant V. I. 2003, Z. Angew. Math. Mech., 83, 363

Fried D. L. 1966, J. Opt. Soc. Am., 56, 1372

Fried D. L. 1978, J. Opt. Soc. Am., 68, 1651

Gradstein I., Ryshik I. 1981, Summen-, Produkt- und Integraltafeln, Harri Deutsch, Thun

Hou X., Wu F., Yang L., Chen Q. 2006, Appl. Opt., 45, 8893

Hu P. H., Stone J., Stanley T. 1989, J. Opt. Soc. Am., A, 6, 1595

Landau L. J., Luswili N. J. 2001, J. Comp. Appl. Math., 132, 387

Lundström L., Unsbo P. 2007, J. Opt. Soc. Am. A, 24, 569

Mahajan V. N. 1981, J. Opt. Soc. Am., 71, 75

Mahajan V. N., Dai G.-m. 2007, J. Opt. Soc. Am., A, 24, 2994

Mathar R. J. 2009, arXiv:0911.4710 [astro-ph.IM]

Mathar R. J. 2010, Waves Random Complex Media, 20, 23

Menut J.-L., Valat B., Lopez B., Schmider F.-X., Vakili F., Jankov S., Bresson Y., Lagarde S., Petrov R. G., Domiciano A., Mosoni L., Danchi W. C. 2008, ApJ, 686,1514

Miller A. R. 1997, J. Comp. Appl. Math., 85, 271

Noll R. J. 1976, J. Opt. Soc. Am., 66, 207

Novario P. G. 2005, Electr. Trans. Num. Anal., 20, 198

Prata A. Jr., Rusch W. V. T. 1989, Appl. Opt., 28, 749

Roddier C., Roddier F. 1976, J. Opt. Soc. Am., 66, 580

Roddier F. 1981, Prog. Opt., 19, 281

Roddier N. 1990, Opt. Eng., 29, 1174

Sabatke E. E., Burge J. H., Hinz P. 2006, Appl. Opt., 45, 8026

Santarsiero M., Gori F., Borghi R., Guattari G. 2007, J. Opt. A: Pure and Appl. Optics, 9, 593

Schwiegerling J. 2002, J. Opt. Soc. Am., A, 19, 1937 
Sheppard C. J. R., Campbell S., Hirschhorn M. D. 2004, Appl. Opt., 43, 3963 Shu H., Luo L., Han G., Coatrieux J.-L. 2006, J. Opt. Soc. Am., A, 23, 1960 Slater L. J. 1966, Generalized Hypergeometric Functions, Cambridge University Press

Swantner W., Chow W. W. 1994, Appl. Opt., 33, 1832

Swantner W. H., Lowrey W. H. 1980, Appl. Opt., 19, 161

Takato N., Yamaguchi I. 1995, J. Opt. Soc. Am., A, 12, 958

Upton R., Ellerbroek B. 2004, Opt. Lett., 29, 2840

Wang J. Y., Silva D. E. 1980, Appl. Opt., 19, 1510 